\title{
Signal detectability in the presence of monotic or dichotic noise bands of equal or unequal levels
}

\author{
J. A. STILLMAN and R. J. IRWIN \\ University of Auckland, Auckland, New Zealand
}

\begin{abstract}
The application of the power-spectrum model of masking to the detectability of a signal masked by dichotic noise was investigated in three experiments. In each experiment, the signal was a $2-\mathrm{kHz}$ sinusoid of $400-\mathrm{msec}$ duration, masked by either one or two $800 \cdot \mathrm{Hz}$ wide bands of noise presented singly or in pairs. In Experiment 1, we compared the detectability of a diotic signal masked by dichotic noise with the detectability of a monaural signal masked by each of the noises separately. The spectrum level of the noise was $35 \mathrm{~dB}$ SPL. For dichotic presentations, the signal was sent to both ears while pairs of noise bands, one below and one above the signal frequency, were presented together, one band to each ear. Threshold levels with the dichotic stimuli were lower than or equal to the thresholds with either ear's stimulus on its own. Similar dichotic stimuli were used in Experiment 2, except that the signal frequency was nearer to one or the other of the bands of masking noise, and the noise had a spectrum level of $50 \mathrm{~dB}$ SPL. In Experiment 3 , thresholds were obtained with two sets of symmetrically and asymmetrically placed notched-noise maskers. For one of these sets, the spectrum level of both noise bands was $35 \mathrm{~dB}$ SPL; for the other set, interaural intensity differences were introduced in the form of an inequality in the levels of the noise bands on either side of the signal. In one ear, the spectrum level of the lower frequency noise band was $35 \mathrm{~dB}$ SPL and the spectrum level of the higher frequency noise band was $25 \mathrm{~dB}$ SPL, whereas in the other ear, the allocation of noise level to noise band was reversed. The dichotic thresholds obtained with the unequal noise maskers could be predicted from the shapes of the auditory filters derived with equal noise maskers. The data from all three experiments suggest that threshold signal levels in the presence of interaural differences in masker intensity depend principally on the ear with the higher signal-to-masker ratio at the output of its auditory filter, a finding consistent with the power-spectrum model of masking.
\end{abstract}

The outcomes of numerous psychophysical and physiological studies suggest that the analysis performed by the peripheral auditory system can be modeled by a bank of overlapping band-pass filters. The attenuation characteristic or shape of the auditory filter has been revealed through the analysis of threshold data from a number of experiments. These studies show that at intermediate frequencies (between about 1 and $7 \mathrm{kHz}$ ) and at moderate sound levels (below a spectrum level of about $40 \mathrm{~dB}$ SPL), the bandwidth of the hypothetical filter is approximately proportional to its center frequency, and comparable attenuation is applied to frequencies located at equal distances on either side of the filter center (Moore \& Glasberg, 1983). With increasing sound level, however, the bandwidth and asymmetry of the filter tend to increase (Glasberg, Moore, Patterson, \& Nimmo-Smith, 1984; Lutfi \& Patterson, 1984; Pick, 1980; Weber, 1977).

We wish to thank Peter F. Jenkins for the loan of some equipment and Stuart Smith for technical assistance. Experiment 3 was supported by a grant-in-aid from the Auckland University Research Committee. This article was significantly improved over an earlier version through the constructive comments of an anonymous reviewer. Correspondence may be addressed to J. A. Stillman, now at the Department of Psychology, University of Otago, P.O. Box 56, Dunedin, New Zealand.
A single underlying model of masking has been applied to the analysis of data from experiments involving a diversity of maskers (see Glasberg \& Moore, 1982, 1984). These maskers include, for example, tonal maskers (Patterson \& Henning, 1977) and a variety of noise maskers, including high- or low-pass filtered noise (Margolis \& Small, 1975; Patterson, 1974), rippled noise (Houtgast, 1974, 1977; Pick, 1980; Yost, 1982), and symmetric and asymmetric notched noise (Glasberg et al., 1984; Moore \& Glasberg, 1981; Patterson, 1976; Patterson \& NimmoSmith, 1980; Patterson, Nimmo-Smith, Weber, \& Milroy, 1982; Tyler, Hall, Glasberg, Moore, \& Patterson, 1984; Weber, 1977). This model assumes that, for a specified level of performance in a detection task, a constant ratio of signal power to masker power is required at the output of the filter. Ordinarily, it is assumed that a detector following the filter monitors whichever member of the bank of filters yields the largest ratio of output signal power to output masker power. Thus, depending on the spectrum of the masker and the shape of the filter, detection may not be determined by the output of a filter centered on the signal (Patterson, 1976; Patterson \& Nimmo-Smith, 1980).

The auditory stimulus in normal listening, in contrast to that usually employed in auditory-filter experiments, 
is subject to interaural temporal and intensive differences, and it is unclear whether the effective masker for the power-spectrum model of masking is a combination of the masking sounds in each ear, or whether detection is governed by the ear affording the better signal-to-masker ratio at the output of the auditory filter. The experiments reported here address this question in the limited circumstance in which interaural intensity differences are present, but interaural temporal differences are absent.

\section{EXPERIMENT 1}

The purpose in Experiment 1 was to compare the detectability of a signal that is masked dichotically-that is, by a different noise in each ear-with the detectability of the same signal when it is masked by each of the constituent masking noises separately. In this way, the independent effect of each masking noise can be determined. In accordance with a power-spectrum model of masking, when a single band of noise or a notched noise is progressively distanced from the signal, decreases in signal level for constant detectability keep pace with reductions in the noise passed by the auditory filter. In Experiment 1, threshold curves obtained with a band of filtered noise positioned either below or above the signal were compared with a threshold curve obtained with dichotic presentations of both of these stimuli simultaneously. The between-the-ears segregation of the masking frequencies with the dichotic stimuli amounts to an extreme interaural intensity difference, wherein the frequencies present in one ear are subject to a practically infinite attenuation in the other.

\section{Method}

A set of four threshold curves was obtained from each of 2 listeners. Three of these curves were obtained with monotic stimuli when the signal and the masker were presented together to only one ear, and a fourth threshold curve was obtained with dichotic stimuli. On all occasions, the signal was a 400 -msec sinusoid, $f_{0}=2 \mathrm{kHz}$, masked by either one or two $800-\mathrm{Hz}$ wide bands of noise at various spectral locations. The masking noise was on continuously throughout each block of trials, at a spectrum level of $35 \mathrm{~dB}$ SPL. Two of the three monotic threshold curves were generated with a single band of masking noise located either below or above the signal frequency, and the third was generated when pairs of lower and upper frequency noise bands were presented together so as to form a spectral notch centered on the signal. For the remaining threshold curve, the pairs of lower and upper frequency masking noises were used once more, but this time, the lower frequency noise band was sent to one ear and the upper frequency noise band was sent to the other ear. For this dichotic presentation, the signal was sent in phase to both ears. The same separations, $\Delta f / f_{0}$, between the signal and the nearer edge of one noise band were used with each set of thresholds. The values of $\Delta f / f_{0}$ were $0,0.075$, $0.125,0.175,0.275$, or 0.375 .

Generation and control of stimuli. The $400-\mathrm{msec}$ analog signal was gated with $100-\mathrm{msec}$ linear onset and offset ramps. A computer controlled the signal presentation in conjunction with a programmable attenuator (Charybdis, Model D), recorded the listener's response, and calculated threshold values. Each sharpedged band of masking noise was generated by a modulation tech- nique (Greenwood, 1961; Patterson, 1976) as follows: A broadband noise produced by a General Radio noise generator (Model 1381) was low-pass filtered (Wavetek Model 852), so that the slope of the noise beyond the cutoff was $96 \mathrm{~dB}$ per octave. The cutoff frequency at the half-power point was $400 \mathrm{~Hz}$. The filtered low-pass noise was then sent to a specially constructed pair of double balanced modulators (Plessey SL 1640C), in each of which it was multiplied with a different sinusoid (or carrier), to produce two bands of noise each $800 \mathrm{~Hz}$ wide, extending from the carrier frequency to $\pm 400 \mathrm{~Hz}$. The band of noise above each carrier is a frequency-shifted replica of the power spectrum of the low-pass noise, while that below each carrier frequency is its mirror image. Carrier suppression was minus $40 \mathrm{~dB}$, relative to the spectrum level of the noise at the output of the modulators. Intermodulation products were removed by bandpass filtering (Khron-Hite Model 3550R). A remnant of the 2 nd harmonic of each carrier remained after this process; it was removed by subsequent low-pass filtering (Kemo VBF/8). To facilitate precise control of the spectrum levels, each band of noise was routed through an amplifier (Hewlett-Packard 467A) and an attenuator (Marconi TF 2162), before being mixed with the signal. Calibration and monitoring of stimulus levels were performed with the aid of a Hewlett-Packard spectrum analyzer (Model 3582A) and an artificial ear (Brüel and Kjaer, Model 4152) with a 6-cc coupler.

Procedure. Each threshold estimate was the average of at least two blocks of trials, and between the two estimates of a threshold the placement of the headphones was reversed, so that both monotic and dichotic thresholds are the average of the two ears. If the two estimates contributing to a monotic threshold differed by more than $3 \mathrm{~dB}$, the pair of trial blocks was repeated, and all four estimates were averaged to give a final value. Dichotic thresholds differing by more than $5 \mathrm{~dB}$ were also repeated.

A two-alternative forced-choice procedure was used to obtain an estimate of the signal level required for $80 \%$ correct detection. Threshold values were obtained by means of the adaptive psychophysical procedure, PEST (parameter estimation by sequential testing; Taylor \& Creelman, 1967). The deviation limit was 1 , and the procedure stopped when a $0.5-\mathrm{dB}$ step was called for. The initial stimulus levels for each block were determined for the subjects individually, on the basis of performance in practice sessions. The initial level was chosen to be approximately $15 \mathrm{~dB}$ above threshold.

A trial began with a 500 -msec warning interval, followed by two 400 -msec observation intervals. The three intervals, marked by a succession of lights, were each separated by $500 \mathrm{msec}$. The task was self-paced, and, after each response, a 300 -msec light indicated the interval in which the signal had occurred. Each subject was given $3 \mathrm{~h}$ of practice before data were collected, and experimental sessions lasted between 1 and $2 \mathrm{~h}$, with the longer sessions including a 15 -min break.

Subjects. The subjects were 2 women, R.C. and J.S. (one of the authors). Neither subject had a loss of more than $15 \mathrm{~dB}$ (re ISO standards) in either ear at any of seven audiometric frequencies, when tested with a Brüel and Kjaer automatic audiometer (Model 1800). One subject, J.S., had previous experience in psychoacoustic tasks.

\section{Results}

The data from the experiment are displayed separately for each listener in Figure 1. Threshold signal level for $80 \%$ correct detection is shown as a function of the normalized distance $\left(\Delta f / f_{0}\right)$ from the signal to the edge of one noise band.

Within each panel, the top three curves, beginning with the upper curve, show monotic thresholds with, respectively, a notched noise, a single band of noise below the signal, and a single band of noise above the signal. The bottom curve shows thresholds with a dichotic masking 


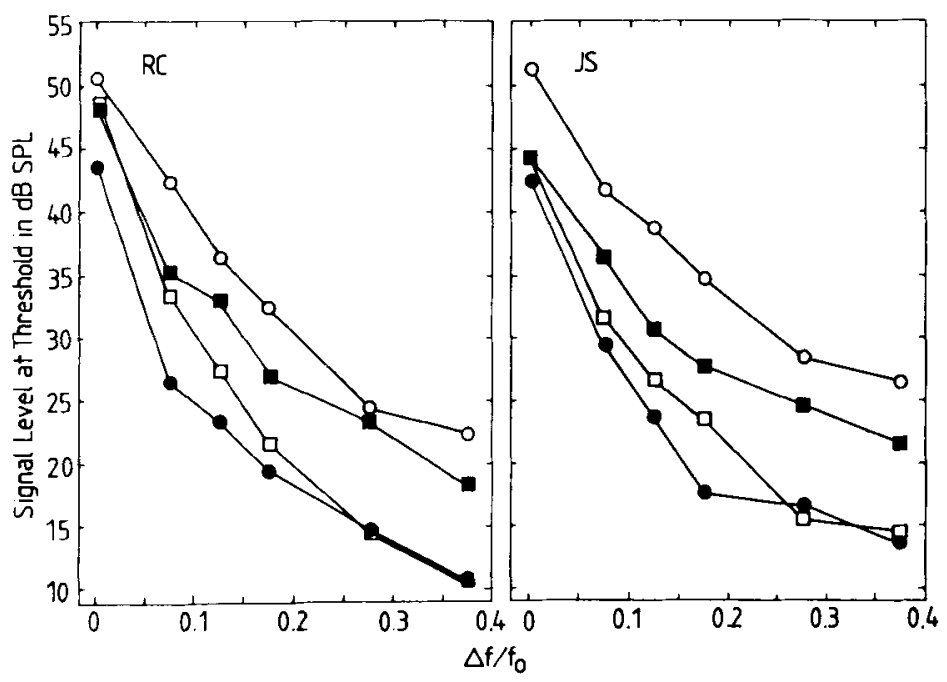

Figure 1. Signal level at threshold for two listeners, R.C. and J.S., as a function of the normalized distance, $\Delta f / f_{0}$, from the $2-\mathrm{kHz}$ signal to the edge of one noise band, with a symmetrically positioned notched noise (open circles), a single band of noise below the signal frequency (filled squares), a single band of noise above the signal frequency (open squares), or a dichotic presentation of both noise bands simultaneously, one in each ear (filled circles). The spectrum level of the masking noise was $35 \mathrm{~dB}$ SPL.

noise-that is, when the diotic signal was masked by the lower frequency noise in one ear, and the higher frequency noise in the other ear.

The threshold curves generated with a notched noise are representative of results from this type of experiment. When one or the other of the masking noises on either side of the notch was omitted, the resulting threshold curves were displaced downwards. The average reductions in signal level relative to thresholds with a notched noise were $4.0 \mathrm{~dB}$ for R.C. and $6.0 \mathrm{~dB}$ for J.S. with the low-pass noise, and $8.7 \mathrm{~dB}$ for R.C. and $10.9 \mathrm{~dB}$ for J.S. with the high-pass noise. A lowering of the threshold in excess of $3 \mathrm{~dB}$ consequent upon a $50 \%$ reduction in masker power has been interpreted by Patterson and Nimmo-Smith (1980) as strong evidence of off-frequency listening. For each listener, the separation between the pairs of curves with single-noise maskers (the squares in Figure 1) suggests a degree of filter asymmetry, even at this moderate intensity, with the lower branch of the filter being broader than the upper. The feature of most interest in each panel, however, is the location of the threshold curve generated with a dichotic stimulus relative to the location of the other curves. The net masking with the dichotic stimulus is not an average of the masking in each ear. Instead, signal thresholds with the combination stimulus are generally lower than with the more detectable of the dichotic components, each of which provides a significant opportunity to improve detection by shifting the filter off-frequency. In other words, dichotic thresholds are even lower than those for the better ear (the ear with the higher signal-to-masker ratio at the output of its auditory filter).
Two phenomena need to be considered in relation to the outcomes of this experiment. They are comodulation masking release (CMR) and the binaural masking-level difference (BMLD). The possible relevance of CMR to this study arises because whenever two noise bands were used they were produced by multiplying a single low-pass noise with two different sinusoids. The two noise bands were therefore comodulated, having temporal envelopes in common. We think it unlikely, however, that CMR effects were responsible for the improved detection of our dichotic stimuli. This is because CMR effects have so far been demonstrated only for signals centered in one of the masking bands (see, e.g., Hall, Haggard, \& Fernandes, 1984), whereas our signal was located in a spectral gap between a pair of masking bands. Furthermore, by contrast with the outcome of Experiment 1, a dichotic CMR is in general smaller than a monotic CMR (Schooneveldt \& Moore, 1987). With respect to a BMLD, the major features usually associated with this phenomenon are absent from the dichotic stimuli used here, and mechanisms associated with it are unlikely to be responsible for the improvement for dichotic thresholds over thresholds obtained from the better ear. BMLDs generally occur when either the phase or level differences of the signal at the two ears are different from those of the masker (Moore, 1982, p. 193). Our dichotic stimuli involved no overall intensity differences between the ears. The total noise power was the same in each ear, and the diotic $2-\mathrm{kHz}$ signal was above the frequency for which interaural time differences are able to be utilized. Furthermore, in a variety of experiments concerned with BMLDs surveyed by Durlach 
and Colburn (1978), the masking noise always had a relatively large bandwidth, with a power spectrum flat across a critical band centered on the tone. On all but one occasion in our Experiment 1, the signal was spectrally separated from the band of masking noise.

A number of possibilities can be considered to account for the improvement of our dichotic thresholds over the monotic thresholds. For instance, such an improvement could arise on a statistical basis, because, owing to the inherent fluctuations in the noise, the ear with the signal that is on the average more masked may, on occasion, contain the more detectable signal. This possibility is supported by the fact that, for each subject, the improvement lessens as the difference between the pair of threshold curves with the single-noise maskers (the squares) increases. In other words, when a masker in one ear provides a large advantage over the masker in the other ear (as happens at large values of $\Delta f / f_{0}$ ), the probability of this advantage's being offset by fluctuations in the noise is very small. Hence, in these circumstances, the effect of the dichotic masker is identical to that of the masker in the ear that affords the better signal-to-masker ratio at the output of its filter. An additional possibility is that the advantage occurred for the dichotic stimulus because of the use of a diotic rather than a monotic signal, notwithstanding that masking was dominated by the noise in only one of the ears. However, the latter explanation is not compatible with the fact that the improvement in dichotic listening over either monotic presentation depends on the difference between the two monotic thresholds.

In Experiment 2, we tested the hypothesis that dichotic detection depends principally on the ear with the more favorable signal-to-masker ratio at the output of the auditory filter.

\section{EXPERIMENT 2}

\section{Rationale}

The dichotic masking noise used in Experiment 1 consisted of a pair of noise bands on either side of the signal, each presented to only one ear. Each noise band was the same distance from the signal in linear frequency. In Experiment 2 , similar dichotic stimuli were used, except that the one or the other noise band was always $150 \mathrm{~Hz}$ nearer to the signal than the other one was. In monaural listening, when a signal is masked by a noise containing a spectral notch whose center is located either below or above the signal frequency, any asymmetry of the auditory filter determines whether thresholds will be lower when the lower frequency edge of the notch is nearer to the signal, or when the upper frequency edge of the notch is nearer to the signal.

Glasberg et al. (1984) describe the asymmetry of the filter at $45 \mathrm{~dB}$ as an expansion of the frequency scale on the low-frequency side of the filter. At this intensity, therefore, thresholds with a monotic notched noise are found to be lower when the upper frequency edge of the notch is nearer to the signal, because less noise is passed by the filter with this configuration than when the lower frequency edge of the notch is nearer to the signal. By the same reasoning, if the effective masker with dichotic stimuli is a combination of the masking sounds in each ear, results analogous to the monotic case should be obtained if the two noise bands are placed asymmetrically about the signal frequency. That is, lower thresholds should be obtained when the upper frequency noise is nearer to the signal, rather than when the lower frequency noise is nearer to the signal.

In contrast to the monotic case, if detection with the dichotic stimuli depends principally upon the ear with the higher signal-to-masker ratio at the output of the auditory filter, lower thresholds should be obtained when the lower frequency masking noise is nearer to the signal than when the higher frequency masking noise is nearer to the signal. This is because, in the former case (lower frequency noise nearer to the signal), the ear with the better signal-to-masker ratio will almost certainly be the one receiving the higher frequency noise, which not only is more remote from the signal, but also is in any case more effectively eliminated, because the filter's narrow branch is on the high-frequency side. On the other hand, when the higher frequency noise is nearer to the signal, it is not immediately clear which ear would have the better signal-to-masker ratio; the lower frequency noise is more remote, but the higher frequency noise would, as before, be more effectively eliminated by the upper branch of the filter. In any case, neither ear would achieve as high a signal-to-masker ratio as the better ear in the former case. It is probable, therefore, that when the higher frequency noise is nearer to the signal, the ear with the higher signalto-masker ratio in its auditory filter will depend on an interaction between the particular location of the noise bands and the magnitude of the filter asymmetry.

\section{Method}

Stimuli and Procedure. Stimulus generation and procedures were similar to those used with the dichotic stimuli in Experiment 1. As before, the signal was a diotic $2-\mathrm{kHz}$ sinusoid masked in each ear by a different $800-\mathrm{Hz}$ wide band of noise. The spectrum level of the noise was $50 \mathrm{~dB}$ SPL. The masking noise in one ear was below the frequency of the signal, while the masking noise in the other ear was above the frequency of the signal. The normalized distances, $\Delta f / f_{0}$, from the nearer edge of one noise band to the signal were $0,0.05,0.1,0.2,0.3$, and 0.4 , while the remaining noise band was $150 \mathrm{~Hz}\left(\Delta f / f_{0}=0.075\right)$ farther from the signal. Two sets of thresholds were obtained: one when the lower frequency noise was nearer to the signal, and one when the upper frequency noise was nearer to the signal. Threshold in quiet was also measured. Two estimates were obtained with each of the stimuli and averaged. The allocation of the upper and lower frequency noise bands to the left and right ears was reversed between the estimates.

Subjects. Four listeners, 3 women and 1 man, volunteered to act as subjects. One of these, J.S., also participated in Experiment 1. The remaining subjects had no previous experience in psychoacoustic tasks.

\section{Results and Discussion}

All 4 listeners produced a similar pattern of results, and their data have been averaged. The average threshold in 


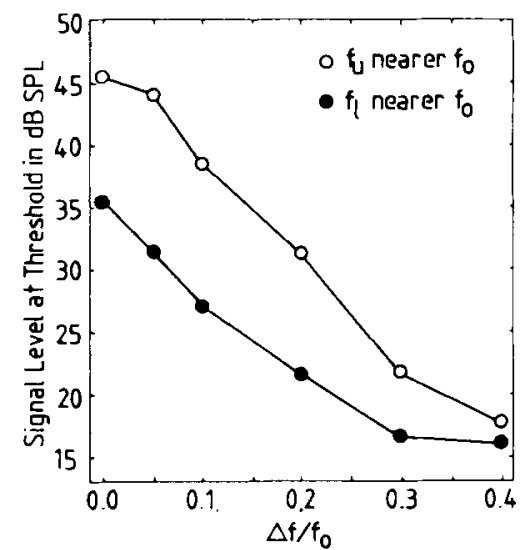

Figure 2. Thresholds of a diotic 2-KHz signal masked by a pair of $800-\mathrm{Hz}$ wide noise bands, one above and one below the frequency of the signal. The masking noises were presented dichotically, one to each ear. The signal level at threshold is shown as a function of the normalized distance, $\Delta f / f_{0}$, from the signal to the nearer edge of whichever masking noise was closer to the signal. The remaining noise band was always $150 \mathrm{~Hz}$ farther from the signal. The spectrum level of the masking noise was $50 \mathrm{~dB}$ SPL, and the data are the average from 4 listeners.

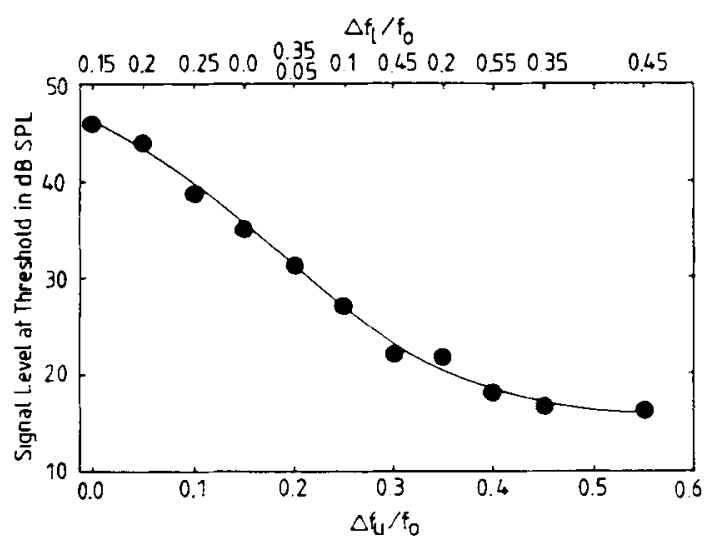

Figure 3. The average thresholds, previously displayed in Figure 2, replotted as a function of the normalized distance $\left(\Delta f / f_{0}\right)$ between the signal frequency and the nearer edge of the upper frequency masking noise. The distance between the signal and the nearer edge of the lower frequency masking noise is given at the top of the figure. A smooth curve has been drawn through the data points.

quiet was $5.0 \mathrm{~dB}$ SPL. Signal levels for $80 \%$ correct detection are displayed in Figure 2, as a function of the normalized distance from the signal to the nearer band of noise.

The relative positions of the two curves demonstrates that the upper frequency noise governed the masking. That is to say, when the upper frequency band was nearer to the signal, the threshold was higher than when the lower frequency band was nearer to the signal. This contrasts with results in monaural studies in which notched-noise or single-noise maskers were used. In monaural studies, the lower frequency noise produces more masking than the upper frequency noise does, indicating that the lower branch of the filter is broader than the upper at this noise level. However, in accordance with our previous reasoning, the dichotic data are consistent with the data from monaural studies, if it is assumed that detection depended on the ear with the higher signal-to-masker ratio at the output of the auditory filter.

At the outset of Experiment 2, it was thought that when the higher frequency noise was nearer to the signal, the ear with the higher output signal-to-masker ratio might vary, depending on an interaction between the asymmetry of the auditory filter and the distances between the signal and each noise band. However, when the data in Figure 2 are plotted as a function of the distance between the signal and the higher frequency noise, the points fall on a single curve. The data, rearranged in this way, are displayed in Figure 3.

The abscissa in Figure 3 gives the systematically increasing distance between the signal and the nearer edge of the upper frequency noise. By contrast, the variable position of the remaining noise band is given at the top of the figure. The smooth curve drawn through the data points displays the characteristic form obtained in monaural studies when the separation between a signal and a masking noise is progressively increased (see Figure 1, squares), exhibiting a decline in the rate at which thresholds fall as the upper frequency noise moves from the skirts to the flatter tails of the filter. The form of this curve implies that the upper branch of the filter passed the lesser quantity of noise even when the upper frequency noise was $150 \mathrm{~Hz}$ nearer to the signal than the lower frequency noise was. In other words, at this noise level, the asymmetry of the auditory filter was sufficiently pronounced so that dichotic thresholds reliably depended on the signalto-masker ratio in the upper branch. There are two points in the figure for which $\Delta f_{u} / f_{0}=0.2$. For one of them, the normalized distance to the lower masking frequencies was 0.05 , and for the other, it was 0.35 . These two points coincide, and thus they demonstrate that, as far as the detectability of the signal was concerned, the spectral location of the lower frequency noise was unimportant.

Dichotic thresholds in Experiment 2, in common with dichotic thresholds in Experiment 1, appear to have been governed principally by the masking in the ear with the higher signal-to-masker ratio at the output of the auditory filter.

\section{EXPERIMENT 3}

In Experiment 3, our goals were: (1) to examine a listener's performance in the presence of a less extreme interaural intensity difference than those applied in the previous experiments; (2) to use a diotic mode of signal presentation throughout the experiment in order to check whether the improvement for dichotic thresholds in Experiment 1 may have resulted from the use of a diotic signal only when dichotic maskers were used and a monotic signal otherwise; and (3) to eliminate the possibility of 
any statistical advantage arising from random differences in the masking noises in each ear. This last goal was achieved by using dichotic maskers with exactly the same phase and frequency composition in each ear.

In this experiment, auditory filter shapes derived from 2 listeners were used to compute theoretical threshold curves for signals masked by the components of the dichotic stimulus present in each ear. The predicted thresholds were subsequently compared to the thresholds actually obtained with dichotic stimuli.

In the first part of Experiment 3, the technique of Patterson and Nimmo-Smith (1980) was used to derive auditory filter shapes at $2 \mathrm{kHz}$ from 2 listeners. With this technique, threshold data are generated with a masking noise that contains a spectral notch placed both symmetrically and asymmetrically about the signal frequency. The threshold data were fitted to Patterson et al.'s (1982) twoparameter rounded exponential model of the filter.

In the second part of Experiment 3, the threshold measures were repeated, using the same notch specifications as in the first part of the experiment, but this time there were interaural differences in the intensity of some masking frequencies. The intensity differences took the form of an inequality in the levels of the two masking bands, one above and one below the signal frequency. The inequality was opposite in each ear.

These interaural intensity differences ensured that although the signal-to-masker ratio at the input to each ear was the same, on most occasions the output from each ear's filter would be different. On some occasions, the signal-to-masker ratio in the auditory filter would be larger in the ear in which the lower frequencies were more intense, and on other occasions, it would be larger in the ear in which the higher frequencies were more intense. More specifically, when the notch was offset about the signal, the ear with the larger signal-to-masker ratio would usually be that in which the frequencies closest to the signal had the lower spectrum level, but when the notch was symmetrically located about the signal, the ear with the larger signal-to-masker ratio would depend on the asymmetry of the auditory filter. The difference between the signal-to-masker ratios in each ear could be assessed from the shape of the auditory filter.

The same signal presentation was used both when the auditory filters were derived and when the dichotic thresholds were measured. That is, the filters were derived using diotic, rather than the more usual monotic stimuli. Little difference is expected between diotic and monotic masked thresholds (see McFadden, 1975, for a review). Weber (1977) also derived filter shapes using diotic stimuli. The equivalent rectangular bandwidths of the filters derived by Weber did not differ from those derived using only one ear, and they are included in Moore and Glasberg's (1983) summary of auditory filter experiments.

\section{Method}

Thresholds were measured for a gated sinusoidal signal, $f_{0}=$ $2 \mathrm{kHz}$, positioned in a spectral notch between two rectangular bands of masking noise $800 \mathrm{~Hz}$ wide. The edges of the noise bands are defined as their half-power points.

A total of 18 notches were used with both the diotic and the dichotic maskers. The 18 notches were divided into three sets of 6 notches each. In two of these sets, the notch center frequency was displaced $150 \mathrm{~Hz}$ either below or above the frequency of the signal. For one set, therefore, the lower frequency noise band was nearer to the signal than the upper, and for the other set, the upper frequency noise band was nearer. For these two sets, normalized distances, $\Delta f / f_{0}$, from the signal to the nearer edge of the notch, were the same as in Experiment 2. For the third set of notches, the signal was equidistant from the two edges of the notch, and the normalized distances from the signal to either edge were $\Delta f / f_{0}=$ $0,0.075,0.125,0.175,0.275$, and 0.375 . The spectrum level of the masking noise for the diotic presentations used to determine the shape of the auditory filter was $35 \mathrm{~dB}$ SPL; for dichotic presentations, the spectrum level of one noise band in each ear was reduced to $25 \mathrm{~dB} \mathrm{SPL}, 10 \mathrm{~dB}$ less than for the same band in the other ear. ${ }^{1}$ The 10-dB difference between the same frequencies at the two ears represents about the maximum difference that can occur in a free sound field for frequencies up to approximately $4 \mathrm{kHz}$ (Feddersen, Sandel, Teas, \& Jeffress, 1957). The phase, frequency composition, signal-to-masker ratio, and total masker power of each member of a pair of dichotic stimuli was identical. Figure 4 illustrates this arrangement schematically for a representative pair of stimuli.

Stimulus generation and Procedure. The $2-\mathrm{kHz}$ signal and sharped-edged bands of masking noise were generated and controlled as described for Experiment 2. The signal and both noise bands were routed through a pair of mixers to each member of a pair of matched TDH-39 headphones in MX/41AR cushions. For the dichotic presentations, one noise band in each ear was attenuated by $10 \mathrm{~dB}$ before being sent to the mixer

Each threshold was measured according to the same adaptive procedure as in the previous experiments, and as before, each threshold was the average of two estimates. When dichotic maskers were used, the allocation of each headphone to a particular ear was reversed between estimates, so that the left ear's stimulus became the right ear's stimulus for the second estimate, and vice versa. The presentation order of the various notches was randomized, and for each notch, diotic and dichotic thresholds were determined consecutively. The order in which these two types of presentation occurred was changed for each new notch. The subjects were given
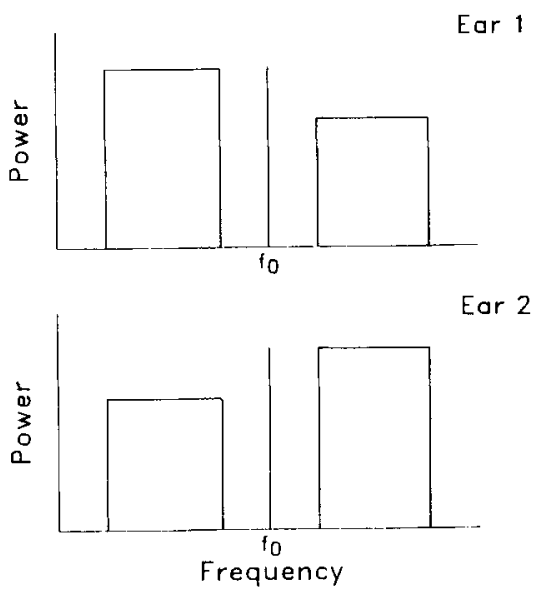

Figure 4. Schematic diagram of a representative pair of dichotic stimuli when the $2-\mathrm{kHz}$ signal was centered in the spectral notch. The spectrum level of the more intense noise band was $35 \mathrm{~dB}$ SPL, while the spectrum level of the less intense noise band was $25 \mathrm{~dB}$ SPL. Each band of noise was $800 \mathrm{~Hz}$ wide. 
$3 \mathrm{~h}$ of practice before data were collected, and experimental sessions lasted between 1 and $2 \mathrm{~h}$. Data were collected from a total of 144 blocks of trials, with an average of 72 trials per block. Pairs of diotic thresholds differing by more than $3 \mathrm{~dB}$ and pairs of dichotic thresholds differing by more than $5 \mathrm{~dB}$ were to be repeated, but with these criteria it was unnecessary to repeat any estimate.

Subjects. The subjects were 2 female undergraduate students at the University of Auckland: B.F., 18 years of age, and K.V., 20 years of age. These listeners were chosen from a number of applicants, and they were paid for their participation. Although it was not necessary for the filters in each ear to be identical, because both diotic and dichotic thresholds were the average from the two ears, we sought to minimize any differences between the ears. Thus, because normal-hearing listeners below 40 years of age in Patterson et al. (1982) displayed only small differences between the two ears' filters, young adult listeners were chosen. In addition, the subjects were selected to have similar audiometric profiles in both ears so as to ensure that the frequency-selective mechanisms operated on stimuli that were subject to comparable attenuations in each ear. ${ }^{2}$

\section{Results}

Auditory filters. In order to derive the auditory filter shapes, each listener's threshold data were fitted to Patterson et al.'s (1982) two-parameter rounded exponential model of the filter. Following the technique described in Patterson and Nimmo-Smith (1980), the data for the three positions of the notch about the signal were fitted to the model concurrently, and individual parameter values were obtained for the lower and upper branches of the filter. The resulting threshold curves are shown, together with the data, in Figure 5. The standard deviation between the observed and predicted threshold levels was $1.3 \mathrm{~dB}$ for B.F. and $1.9 \mathrm{~dB}$ for K.V. At wide notch widths, the fit of the data to the model is less satisfactory for K.V. than for B.F.

The auditory filters derived from the threshold curves for the 2 listeners are shown in Figure 6. As expected, at this moderate sound level, the filters are not markedly asymmetrical. B.F.'s auditory filter is asymmetric in the passband, having a broader lower than upper branch, but the dynamic ranges on the two sides of the filter are similar. K.V.'s auditory filter is symmetrical in the passband, but at $\Delta f / f_{0}=0.4$, the upper branch of the filter provides about $8 \mathrm{~dB}$ more attenuation than the lower.

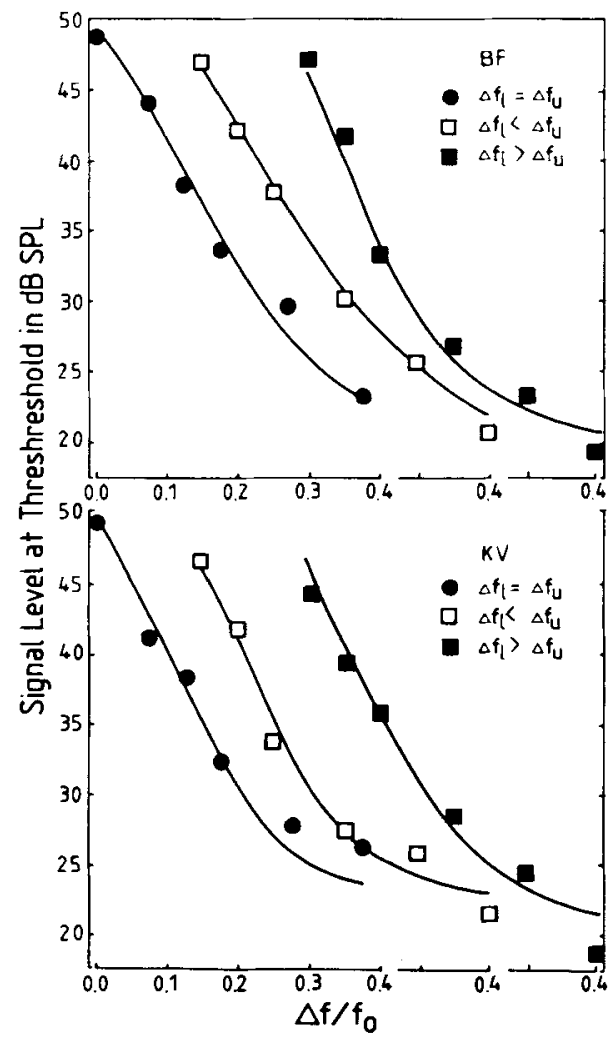

Figure 5. Signal level for $\mathbf{8 0 \%}$ correct detection as a function of the normalized distance, $\Delta f / f_{0}$, from the 2-kHz signal to the nearer edge of a spectral notch in the masking noise. The spectrum level of the masking noise was $35 \mathrm{~dB}$ SPL. For clarity, the origin is displaced 0.15 to the right for each position of the notch about the signal. Symbols are the measured thresholds when the signal was equidistant from each noise band (filled circles), nearer to the lower frequency noise band (open squares), or nearer to the upper frequency noise band (filled squares). The curves are the best-fitting theoretical functions. The data are from 2 listeners.

Thresholds with dichotic stimuli. The relation of the thresholds obtained with dichotic stimuli to the prediction for each ear's stimulus is presented in Figure 7 . The circles show the signal levels for $80 \%$ correct detection obtained with dichotic maskers. In the figure, each column
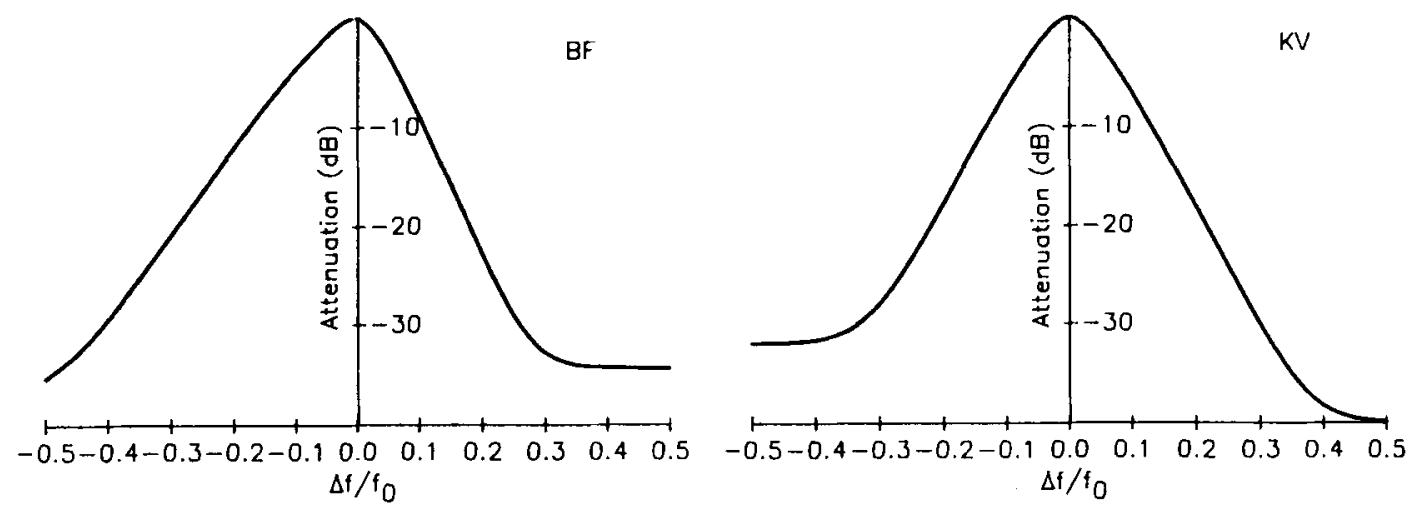

Figure 6. The auditory filters derived from the data in Figure 5 . The filter attenuation in decibels is plotted as a function of the normalized distance from the filter center. 


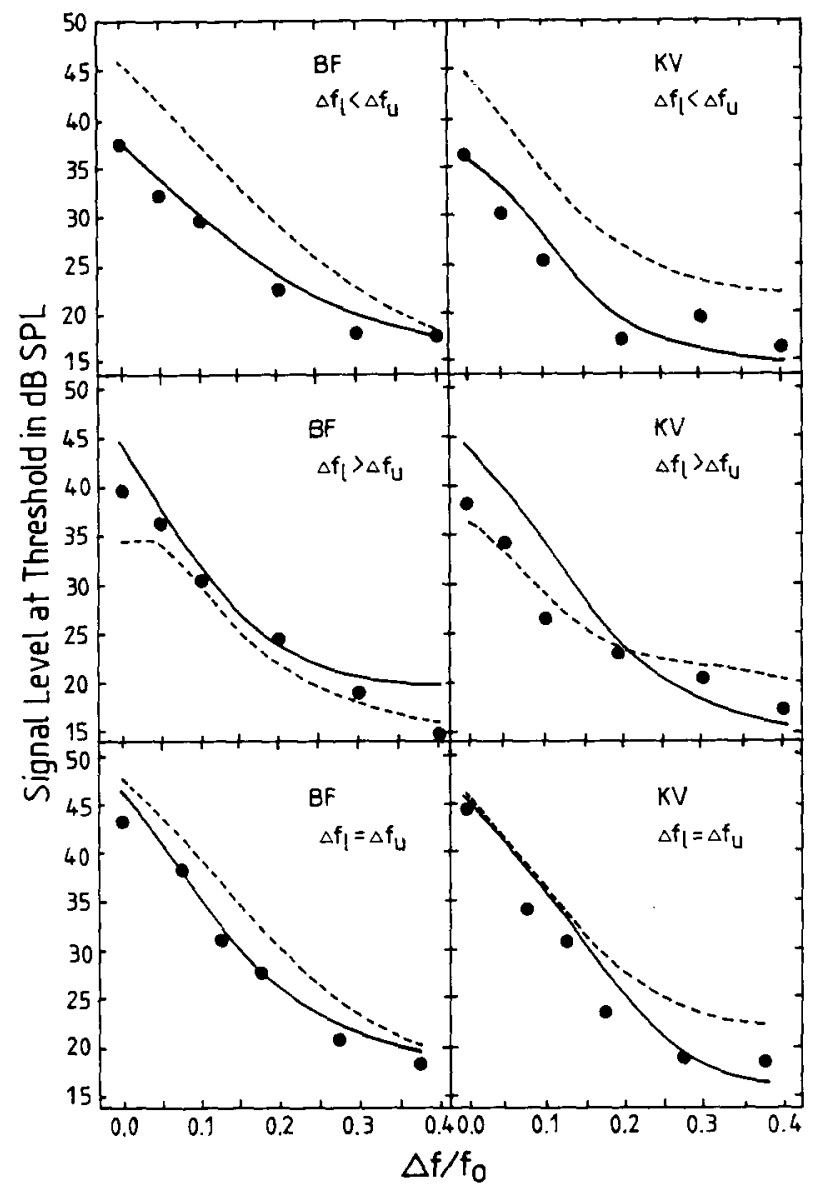

Figure 7. Actual threshold levels (circles) for a diotic 2-kHz sinusoid masked by a pair of noise bands, one below and the other above the signal. The spectral separation of the bands was varied. The masking noise was presented dichotically, and frequency components above and below the signal had unequal spectrum levels. In one ear the spectrum level of the lower frequency noise was 35 dB SPL, whereas the level of the higher frequency noise was $25 \mathrm{~dB}$ SPL, and in the other ear the allocation of noise level to noise band was reversed. The data, for three positions of the signal relative to the nearer edges of the noise, are from 2 listeners, B.F. and K.V. The figure also shows predicted thresholds based on the auditory filter shapes derived previously from each listener (Figure 6). Predictions for the ear in which components of the masker below the signal have the higher spectrum level are represented by the dashed curves, and predictions for the ear in which components of the masker above the signal have the higher spectrum level are represented by the continuous curves. The abscissa of the plots, $\Delta f / f_{0}$, is the normalized distance from the signal to the edge of one of the noise bands. When the signal was ofiset from the center of the spectral gap, $\Delta f / f_{0}$ refers to whichever of the two bands was closer to the signal. In these cases, the remaining noise band was $150 \mathrm{~Hz}$ farther from the signal.

represents a particular listener, and each row represents one of the three positions of the signal, relative to the edges of the notch in the masking noise. The abscissas in the figure give the normalized distance from the signal to the nearer edge of the notch. Except when the signal was centrally located in the notch, the separation between the signal and the more distant edge of the notch was $150 \mathrm{~Hz}$ greater than between the signal and the nearer edge. The dashed curves are the predicted thresholds for an optimally shifted filter when the spectrum level of the masking frequencies below the signal is $35 \mathrm{~dB}$ and the spectrum level of the masking frequencies above the signal is $25 \mathrm{~dB}$. The continuous curves are the predicted thresholds when the masking frequencies above the signal have the higher spectrum level. Each curve is therefore the prediction for the stimulus in one ear, independent of the stimulus in the other ear, and it is based on each listener's auditory filter, derived with diotic stimuli. The ear with the higher signal-to-masker ratio is implicit in the relative position of the two curves in each panel. The relationship between the predictions for each ear changes with notch width and notch offset, reflecting an interaction between the noise levels and the filter asymmetry.

\section{Discussion}

For both listeners, the most reasonable interpretation of the results is that threshold performance with these dichotic maskers is governed principally by the ear with the more favorable signal-to-masker ratio at the output of its auditory filter.

Consider, for example, the top left panel in Figure 7. This panel shows the predictions for subject B.F. when the signal was nearer to the lower frequency noise. The experimental points lie much closer to the continuous curve than to the dashed curve. The continuous curve displays the threshold level predicted from B.F.'s auditory filter (shown in Figure 6) for the ear in which the more distant higher frequency noise band had the greater spectrum level. This panel therefore shows that B.F.'s performance is similar to that expected from the ear with the more favorable signal-to-masker ratio. Similar analyses could be applied to most of the remaining panels of the figure, although when the upper frequency noise is nearer to the signal (the center panels of Figure 4), which prediction best fits the data for B.F. is somewhat equivocal. In general, however, the shape as well as the position of the obtained threshold curves agrees more nearly with the lower than with the upper theoretical curves.

Neither the signal nor the masker in the ear with the lower signal-to-masker ratio appears to have a strong effect on the threshold of the signal. On the average, the thresholds for B.F. in Figure 7 were $0.26 \mathrm{~dB}$ below, and those for K.V. $0.84 \mathrm{~dB}$ below, the prediction for the better ear. For K.V., this average is reduced by the thresholds at the two widest notches, most of which were higher than expected. However, the model of the filter on which the predictions are based failed to describe this listener's data accurately at wide notch widths, and it is notable that the divergence between the theoretical and obtained thresholds has the same form in Figures 5 and 7. These thresholds, therefore, are not inconsistent with the interpretation of the results suggested above.

\section{GENERAL DISCUSSION AND SUMMARY}

In Experiment 1, we compared the detectability of a signal masked by dichotic noise with the detectability of 
a monaural signal masked by each of the noises separately. Threshold levels with the dichotic stimuli were lower than or equal to the thresholds with either ear's stimulus on its own. We investigated this result further in Experiment 2, making use of the fact that with increasing sound levels the lower branch of the filter becomes broader than the upper. A similar dichotic stimulus was used in both experiments, but in Experiment 2, the signal frequency was nearer to one or the other of the bands of masking noise, and the noise had a spectrum level of $50 \mathrm{~dB}$ SPL. The results of both experiments suggested that the thresholds with the dichotic masking noise depended principally on the ear that had the larger signal-to-noise ratio at the output of its auditory filter, a finding consistent with the power-spectrum model of masking. A similar interpretation could be applied to the data of Weston and Miller (1965), who found that if identical tones are presented to the two ears with the signal-to-noise ratio about $25 \mathrm{~dB}$ lower in one ear than in the other, the effect of the signal at the ear with the lower signal-to-masker ratio could be eliminated from the masked threshold. Thresholds in Experiments 1 and 2 , however, were generally lower than those from either ear on its own.

In Experiment 3, thresholds were obtained with maskers subject to less extreme interaural intensity differences than those in the earlier experiments, and the possibility of a statistical advantage for dichotic stimuli was minimized. Perhaps because of this, the improvement for dichotic thresholds over thresholds predicted for the better ear was less than that in Experiments 1 and 2.

Licklider (1948) associated the separation of one sound from other sounds in phenomenal space with an improvement both in the detectability of a tonal signal, and in the intelligibility of speech. We consider it unlikely that this factor significantly improved detection for the dichotic stimuli used in the present study. Although spectral components of the masker either above or below the signal frequency were more intense in one ear than in the other ear, the two bands of noise, when presented together, were not lateralized toward either ear. Listeners were unable to identify separately the maskers in each ear, and they reported that the entire masking noise occupied an area of phenomenal space that enclosed the signal.

In the complex auditory environments of normal listening, interaural temporal and intensive differences are commonplace. These differences play a crucial role in sound localization, and it is well known that interaural phase shifts resulting from differences in the time of arrival of a sound at the two ears can, at some frequencies, produce a useful improvement in detection. With homophasic stimuli, however, Egan, Lindner, and McFadden (1969) found no advantage for two-eared performance. In everyday listening, different sounds are chosen for attention from moment to moment, and these sounds are rarely masked identically in each ear. In these circumstances, the ability of the detector following the filter to discriminate the output of the two ears should be advantageous, because it permits a listener to select the ear with the better signal-to-background ratio and thus effectively disregard the masking sounds in the poorer ear. The ability to give particular attention to one ear's input may underlie the findings of Barroso (1983), who showed that it is easier for a listener to follow one of two competing messages, read by the same speaker, if they are presented dichotically rather than diotically.

\section{REFERENCES}

BARroso, F. (1983). An approach to the study of attentional components in auditory tasks. Joumal of Auditory Research, 23, 157-180. Durlach, N. I., \& ColbuRN, H. S. (1978). Binaural phenomena. In E. C. Carterette \& M. P. Friedman (Eds.), Handbook of perception: Vol. IV. Hearing (pp. 365-466). New York: Academic Press.

Egan, J. P., Lindner, W. A., \& MCFAdden, D. (1969). Maskinglevel differences and the form of the psychometric function. Perception \& Psychophysics, 6, 209-215.

Feddersen, W. E., Sandel, T. T., Teas, D. C., Jeffress, L. A (1957). Localization of high-frequency tones. Joumal of the Acoustical Society of America, 29, 988-991.

GlasberG, B. R., \& MoOre, B. C. J. (1982). Auditory filter shapes in forward masking as a function of level. Journal of the Acoustical Society of America, 71, 946-949.

GlasberG, B. R., Moore, B. C. J. (1984). Comparison of auditory filter shapes derived with three different maskers. Journal of the Acoustical Society of America, 75, 536-544.

Glasberg, B. R., Moore, B. C. J., Patterson, R., \& Nimmo-Smith, I. (1984). Dynamic range and asymmetry of the auditory filter. Journal of the Acoustical Society of America, 76, 419-427.

GreENwOOD, D. D. (1961). Auditory masking and the critical band. Joumal of the Acoustical Society of America, 33, 484-502.

Hall, J. W., Haggard, M. P., a Fernandes, M. A. (1984). Detection in noise by spectro-temporal pattern analysis. Journal of the Acoustical Society of America, 76, 50-56.

HoUTGAST, T. (1974). Lateral suppression in hearing. Unpublished doctoral dissertation, University of Amsterdam.

Houtgast, T. (1977). Auditory-filter characteristics derived from direct masking data and pulsation-threshold data with a rippled-noise masker. Journal of the Acoustical Society of America, 62, 409-415.

LICKLIDER, J. C. R. (1948). Influence of interaural phase relations upon the masking of speech by white noise. Joumal of the Acoustical Society of America, 20, 150-159.

LUtFi, R., PAtterson, R. (1984). On the growth of masking asymmetry with stimulus intensity. Journal of the Acoustical Society of America, 76, 739-745.

MCFAdDEN, D. (1975). Masking and the binaural system. In D. B. Tower (Ed.), The nervous system: Human communication and its disorders (Vol. 3, pp. 137-146). New York: Raven Press.

Margolis, R. H., SMall, A. M. (1975). The measurement of critical masking bands. Journal of Speech \& Hearing Research, 18, 571-587.

MOORE, B. C. J. (1982). An introduction to the psychology of hearing (2nd ed.). New York: Academic Press.

Moore, B. C. J., \& GlasberG, B. R. (1981). Auditory filter shapes derived in simultaneous and forward masking. Joumal of the Acoustical Society of America, 70, 1003-1014.

MoOre, B. C. J., \& Glasberg, B. R. (1983). Suggested formulae for calculating auditory-filter bandwidths and excitation patterns. Journal of the Acoustical Society of America, 74, 750-753.

PAtTerson, R. D. (1974). Auditory filter shape. Joumal of the Acoustical Society of America, 56, 802-809.

Patterson, R. D. (1976). Auditory filter shapes derived with noise stimuli. Joumal of the Acoustical Society of America, 59, 640-654.

Patterson, R. D., \& Henning, B. D. (1977). Stimulus variability and auditory filter shape. Joumal of the Acoustical Society of America, 62, 649-664.

Patterson, R. D., \& Nimmo-Smith, I. (1980). Off-frequency listen- 
ing and auditory-filter asymmetry. Journal of the Acoustical Society of America, 67, 229-245.

Patterson, R. D., Nimmo-Smith, I., Weber, D., \& Milroy, R. (1982). The deterioration of hearing with age: Frequency selectivity, the critical ratio, the audiogram, and speech threshold. Journal of the Acoustical Society of America, 72, 1788-1803.

PICK, G. F. (1980). Level dependence of psychophysical frequency resolution and auditory filter shape. Journal of the Acoustical Society of America, 68, 1085-1095.

SChOONEVELDT, G. P., \& Moore, B. C. J. (1987). Comodulation masking release (CMR): Effects of signal frequency, flanking-band frequency, masker bandwidth, flanking-band level, and monotic versus dichotic presentation of the flanking band. Joumal of the Acoustical Society of America, 82, 1944-1956.

Taylor, M. M., Creelman, C. D. (1967). PEST: Efficient estimates on probability functions. Journal of the Acoustical Society of America, 41, 782-787.

Tyler, R., Hall, J., Glasberg, B., Moore, B., \& Patterson, R. (1984). Auditory filter asymmetry in the hearing impaired. Journal of the Acoustical Society of America, 76, 1363-1368.

WEBER, D. L. (1977). Growth of masking and the auditory filter. Joumal of the Acoustical Society of America, 62, 424-429.

Weston, P. B., Miller, J. D. (1965). Use of noise to eliminate one ear from masking experiments. Journal of the Acoustical Society of America, 37, 638-646.
Yost, W. A. (1982). The dominance region and ripple noise pitch: A test of the peripheral weighting model. Journal of the Acoustical Society of America, 72, 416-425.

\section{NOTES}

1. At moderate noise levels filter asymmetry is generally small. When Lutfi and Patterson (1984) studied the growth of masking asymmetry with stimulus intensity, little change was evident in the asymmetry of masking between the 25- and 35-dB SPL masker levels when the signal was either a 1 - or a $4-\mathrm{kHz}$ sinusoid. Thus, with the noise levels used in this experiment, the filter through which the unequal noise was processed was unlikely to be markedly different from that determined using the diotic maskers.

2. Because the right-ear stimulus for the first estimate of a dichotic threshold became the left-ear stimulus for the second estimate, an examination of the differences between the two estimates should reveal any major differences between the two ears' filters. Over the 18 dichotic thresholds, the average differences between two estimates of a threshold (threshold level at each notch when the higher frequency components had the greater spectrum level in the right ear, minus the threshold level when the higher frequency components had the greater spectrum level in the left ear) were $1.5 \mathrm{~dB}$ for B.F. and $1.3 \mathrm{~dB}$ for K.V. By comparison, over the 18 notches, the average differences between two estimates of a diotic threshold were $0.9 \mathrm{~dB}$ for B.F. and $1.0 \mathrm{~dB}$ for $\mathrm{K}$.V.

(Manuscript received November 21, 1988;

revision accepted for publication September $25,1989$. 